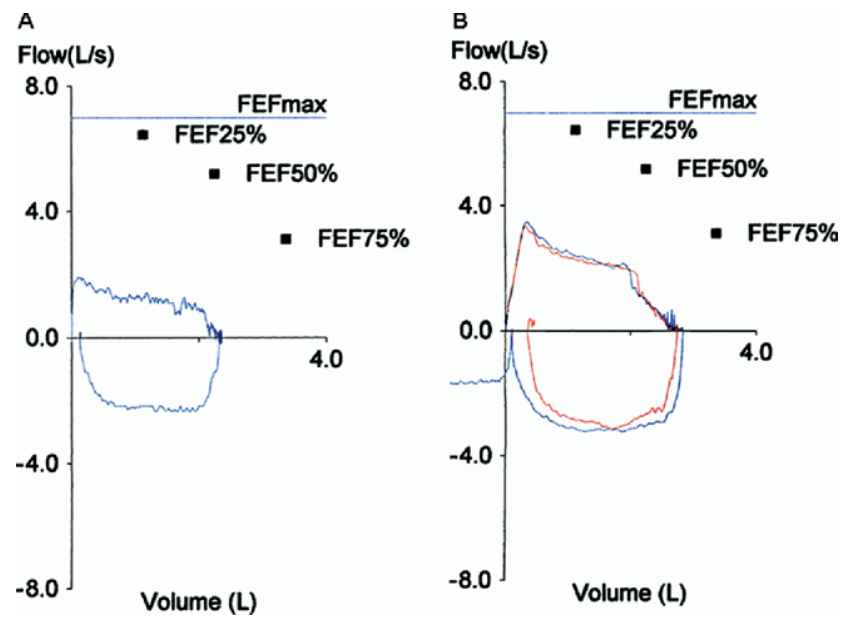

Figure 2. Pulmonary function testing. A, Preoperative flow-volume loop showing significant obstruction. B, Postoperative flow-volume loop demonstrating decreased obstruction. FEF, Forced expiratory flow.

Adoption of less-invasive surgical techniques may improve outcomes by decreasing morbidity, shortening hospital stay, and providing a more desirable cosmetic result, particularly when comparing thoracotomy with thoracoscopy. However, it remains imperative that the fundamental principles that apply to an open approach are followed when performing minimally invasive surgery. In the case of mediastinal repositioning, key points are thorough lysis of adhesions to permit mobilization of the mediastinal structures, placement of prostheses to maintain mediastinal position, and intraoperative bronchoscopic verification of improved airway patency. Here we strictly adhered to these principles. We demonstrated that it is feasible to perform mediastinal repositioning for postpneumonectomy syndrome by using a minimally invasive approach.

\section{References}

1. Adams HD, Junod F, Aberdeen E, Johnson J. Severe airway obstruction caused by mediastinal displacement after right pneumonectomy in a child. A case report. J Thorac Cardiovasc Surg. 1972;63: 534-9.

2. Grillo HC, Shepard JA, Mathisen DJ, Kanarek DJ. Postpneumonectomy syndrome: diagnosis, management, and results. Ann Thorac Surg. 1992; 54:638-50; discussion 650-1.

3. Shamji FM, Deslauriers J, Daniel TM, et al. Postpneumonectomy syndrome with an ipsilateral aortic arch after left pneumonectomy. Ann Thorac Surg. 1996;62:1627-31.

4. Mehran RJ, Deslauriers J. Late complications. Postpneumonectomy syndrome. Chest Surg Clin North Am. 1999;9:655-73.

\title{
Primary pulmonary malignant schwannoma with extension to the tracheal carina
}

Mika Uchiyama, MD, ${ }^{\text {a }}$ Yoshie Shimoyama, MD, ${ }^{\mathrm{b}}$ Noriyasu Usami, MD, ${ }^{\text {a }}$

Simon Ito, MD, ${ }^{a}$ Ayuko Yasuda, MD, ${ }^{a}$ Koji Kawaguchi, MD, and

Kohei Yokoi, MD, ${ }^{\text {a Nagoya, Japan }}$

$\mathrm{P}$ rimary pulmonary malignant schwannomas are extremely rare, and only 11 case reports of this tumor are available. ${ }^{1}$ All cases were treated with a relatively simple surgical procedure, such as pneumonectomy, lobectomy, or enucleation, but their clinicopathologic appearances have not

aFrom the Division of Thoracic Surgery, Department of Surgery, Nagoya University Graduate School of Medicine, ${ }^{\text {a }}$ and the Department of Pathology and Clinical Laboratories, Nagoya University Hospital, ${ }^{\mathrm{b}}$ Nagoya, Japan.

Received for publication July 21, 2006; accepted for publication Sept 5, 2006.

Address for reprints: Mika Uchiyama, MD, Division of Thoracic Surgery, Department of Surgery, Nagoya University Graduate School of Medicine, 65 Tsurumai-cho, Showa-ku, Nagoya 466-8550, Japan (E-mail: takepon@ med.nagoya-u.ac.jp).

J Thorac Cardiovasc Surg 2007;133:265-7

$0022-5223 / \$ 32.00$

Copyright (C) 2007 by The American Association for Thoracic Surgery doi:10.1016/j.jtcvs.2006.09.004 been well clarified. We present a patient with this tumor, which originated in the right upper lobe of the lung and involved the main-

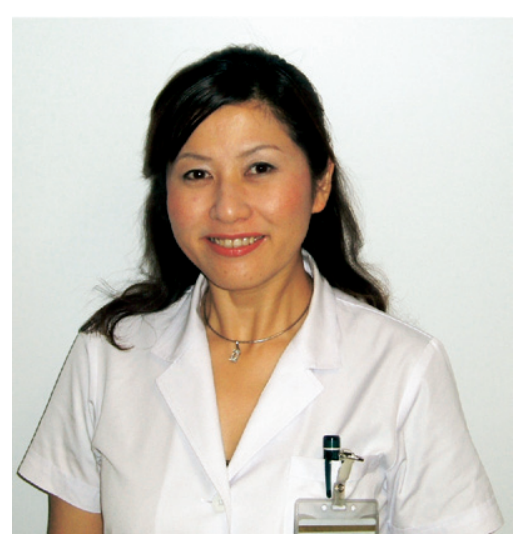

Dr Uchiyama stem bronchus and tracheal carina. For complete removal of the tumor, we performed right upper lobectomy of the lung using extended wedge resection of the tracheal carina, followed by anastomosis of the right intermediate trunk and the distal trachea.

\section{Clinical Summary}

A 57-year-old woman was admitted to our hospital with a 5-month history of worsening cough and dyspnea. There were no abnormal findings on physical and laboratory examinations. Chest x-ray films and computed tomographic scans revealed an apical rightsided large mass measuring $8.4 \times 6.0 \mathrm{~cm}$, which was adjacent to 


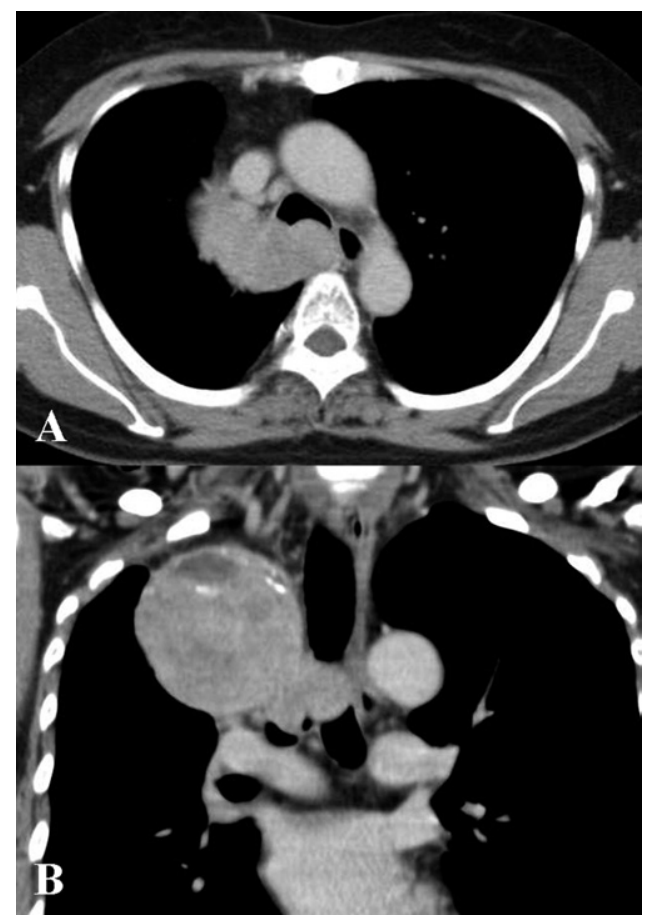

Figure 1. A, Chest computed tomographic scan showing an apical right-sided large mass, which was adjacent to the posterior wall of the distal trachea. B, Computed tomographic scan of the coronal view showing the tumor extended into the right mainstem bronchus as a polypoid lesion across the orifice of the right upper lobe.

the superior vena cava, esophagus, and posterior wall of the distal trachea. The tumor also extended into the right mainstem bronchus as a polypoid lesion across the orifice of the right upper lobe (Figure 1). A bronchoscopic examination disclosed that the distal trachea was decompressed by the tumor from the right dorsal to the left side, and the right main bronchus was almost occluded by the polyp-shaped phyma. Computed tomography-guided percutaneous needle biopsy was applied, and the pathologic diagnosis was schwannoma.

After achievement of general anesthesia with a double-lumen endotracheal tube, the operation was accomplished by means of a right posterolateral thoracotomy approach through the fifth intercostal space. The right upper lobe showed atelectasis with adhesion to the esophagus and superior vena cava, and the whole lobe was almost occupied with the tumor, which extended to the right main bronchus and tracheal carina. The superior pulmonary vein and superior trunk of the pulmonary artery were collapsed. The patient underwent right upper lobectomy of the lung with extended wedge resection of the right main bronchus and tracheal carina, including 3 cartilage rings of the trachea, followed by anastomosis of the right intermediate trunk and the distal trachea. The frozen sections of the surgical margins of the resected trachea and bronchus were negative for malignancy. The prepared fifth intercostal

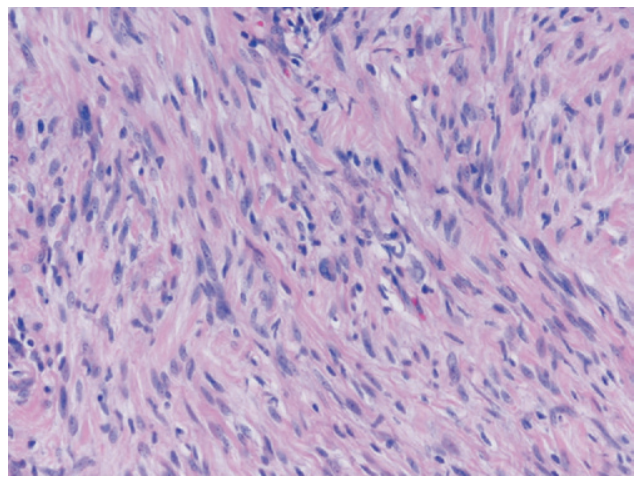

Figure 2. Histologically, the tumor consisted of spindle cells arranged randomly with hyperchromatic and atypical nuclei. (Hematoxylin-eosin stain, original magnification $200 \times$.)

muscle flap was placed between the anastomosis and the pulmonary artery.

Pathologic examination demonstrated that the tumor, which measured $8.0 \times 5.5 \times 4.2 \mathrm{~cm}$, occupied almost all the right upper lobe of the lung and invaded the right main bronchus and tracheal carina. Histologically, the tumor consisted of spindle cells, which were arranged randomly with hyperchromatic and atypical nuclei (Figure 2). There were few findings of nuclear palisading. Immunohistochemical study showed positive staining for S100 protein and negative staining for smooth muscle actin, CD34, neurofilament, and epithelial membrane antigen. There was $5 \%$ to $6 \%$ positive staining for MIB-1 (Ki-67), which indicates low-grade malignancy. Results from histologic and immunohistochemical studies yielded a definitive diagnosis of low-grade malignant schwannoma of the lung. The postoperative course was uneventful. A bronchoscopic examination was performed 7 months after the operation and revealed a well-patent anastomosis without recurrence.

\section{Discussion}

Malignant schwannomas account for approximately $5 \%$ to $10 \%$ of all soft tissue sarcomas. In its classic form a malignant schwannoma arises as a large fusiform or eccentric mass in a major nerve. Most malignant schwannomas are highly invasive and are associated with a low survival rate. ${ }^{2}$ Primary pulmonary malignant schwannomas are extremely rare, and only 11 surgical cases have been reported in the literature. ${ }^{1}$ Those patients were treated with pneumonectomy, lobectomy, or enucleation. One patient with a tumor $4 \mathrm{~cm}$ in diameter underwent a sleeve lobectomy. There were no reports of primary pulmonary malignant schwannoma with extension to the tracheal carina, as in our case. Prognoses of the patients with this tumor were generally poor. Two patients survived more than 20 years, whereas the others died of local recurrence or distant metastasis within 3 years. ${ }^{3,4}$ Because chemotherapy and radiotherapy have not been effective, the curative therapy for this disease is considered to be only radical resection.

Although carinal resection and reconstruction of the airways were used in the management of patients with malignant tumors involving the tracheal carina, the complications of anastomosis 
occurred frequently because of excessive tension, deficiency of blood flow at the site of anastomosis, or both. ${ }^{5}$ We successfully performed right upper lobectomy of the lung using extended wedge resection of the tracheal carina, and this technique was as easy and safe as the tracheobronchoplastic procedure. Furthermore, because of the nature of the tumor without diffuse infiltration, tumor-free surgical margins were obtained in this case.

This is the first case report of primary pulmonary malignant schwannoma involving the tracheal carina, which was successfully removed by using the extended bronchoplastic procedure.

\section{References}

1. Togashi K, Hirahara H, Sugawara M, Oguma F. Primary malignant schwannoma of the lung. Jpn J Thorac Cardiovasc Surg. 2003;51:692-5.

2. Wong WW, Hirose T, Scheithauer BW, et al. Malignant peripheral nerve sheath tumor: analysis of treatment outcome. Int $J$ Radiat Oncol Biol Phys. 1998;42:351-60.

3. Janssen JP, Mulder JJ, Wagenaar SS, et al. Primary sarcoma of the lung: a clinical study with long-term follow-up. Ann Thorac Surg. 1994;58:1151-5.

4. Keel SB, Bacha E, Mark EJ, et al. Primary pulmonary sarcoma: a clinicopathologic study of 26 cases. Mod Pathol. 1999;12:1124-31.

5. Grillo HC. Carinal reconstruction. Ann Thorac Surg. 1982;34:356-73.

\title{
Video-assisted mediastinoscopy improved radical resection for cancer in transhiatal esophagectomy
}

\author{
Daniel Pop, MD, Nicolas Venissac, MD, and Jerome Mouroux, MD, Nice, France
}

T ranshiatal esophagectomy (THE), popularized by Orringer and Sloan, ${ }^{1}$ was proposed to decrease postoperative morbidity and mortality. The major inconvenience is the mediastinal lymphadenectomy that guarantees radical oncologic surgery. Recently, Bumm and associates $^{2}$ used an endodissector that eliminated the "blind" mediastinal dissection. Furthermore, the advent of video-assisted technology provided increasing visualization and allowed bimanual maneuvers. ${ }^{3,4}$ This is our preliminary report using videoassisted mediastinoscopy (VAM) during THE, including technical details.

\section{Patients and Techniques}

Between October 1, 2001 and January 31, 2003 (a 15-month period), we operated on our first 5 patients. The usual pretherapeutic staging and preoperative assessment were done. One patient had neoadjuvant chemoradiotherapy. All 5 patients' characteristics are noted in Table 1 .

The surgical procedures were done by the same team (surgeon with two assistants). The patients were supine with one roll beneath the scapulae (for maximal cervical extension). The abdominal and cervical phases were done by the standard technique. ${ }^{1}$ Care must be taken to avoid arrhythmia or hypotension because of cardiac displacement during abdominal upward dissection of the esophagus. The mediastinal phase is done

From the Thoracic Surgery Department, Pasteur Hospital, Nice, France.

Received for publication June 24, 2006; revisions received Aug 10, 2006; accepted for publication Aug 25, 2006.

Address for reprints: Daniel Pop, MD, Thoracic Surgery Department, Pasteur Hospital-Building H1, 30 Avenue de la Voie Romaine, 06002 Nice, France (E-mail: danielpopch@yahoo.com).

J Thorac Cardiovasc Surg 2007;133:267-8

$0022-5223 / \$ 32.00$

Copyright $\odot 2007$ by The American Association for Thoracic Surgery doi:10.1016/j.jtcvs.2006.08.066 by VAM only through a cervicotomy (Figure 1). The equipment and the instruments have been previously described. ${ }^{4}$ First, along the left side of the esophagus, the video-mediastinoscope allows clear visualization of the left recurrent nerve to the aortopulmonary window level. With bimanual blunt dissection and hemostatic clips, the lymph nodes situated next to the nerve can be easily separated without hurting the nerve. The video-mediastinoscope can be pushed farther into the mediastinum until the full length is reached. Next the anterior side is dissected. The back surface of the trachea, the carina, and the lymph nodes can be safely detached. Then the posterior side of the esophagus can be easily dissected from the prevertebral fascia. The thoracic duct can be identified and endoclips can be used generously for lymphostasis. Finally, the right side of the esophagus can be dissected by passing behind or in front of the esophagus, avoiding injury to the azygos system. Normally, the mediastinal pleurae are not opened. When the superior mediastinal phase is accomplished, the video-mediastinoscope provides the light to reach the upward abdominal dissection of the esophagus. We prefer a gastric substitute. One patient needed a left colon graft because of total gastrectomy for synchronous antral cancer, which lengthened the intervention. The mean operative time for the remainder was about 280 minutes. The mean

TABLE 1. Patients' characteristics

\begin{tabular}{lccccc}
\hline $\begin{array}{l}\text { No. (age [y], } \\
\text { sex) }\end{array}$ & $\begin{array}{c}\text { IT/length } \\
\text { (cm) }\end{array}$ & \multicolumn{1}{c}{ uTN/pTN } & FEV $_{\mathbf{1}}(\mathbf{L} / \%)$ & $\begin{array}{c}\text { Surgery } \\
(\mathbf{m i n})\end{array}$ & $\begin{array}{c}\text { Survival } \\
(\mathbf{m o})\end{array}$ \\
\hline $1(73, \mathrm{~F})$ & $29 / 2$ & T1 N1/T1 N0 & $2.2(130)$ & 220 & $42-\mathrm{AWD}$ \\
$2(70, \mathrm{M})$ & $25 / 5$ & T3 N0/T3 N0 & $1.5(54)$ & 330 & $37-\mathrm{AWD}$ \\
$3(51, \mathrm{M})$ & $27 / 6$ & T3 N1/T3 N1 & $2.7(91)$ & 480 & $17-\mathrm{DWD}$ \\
$4(76, \mathrm{~F})$ & $31 / 2$ & T2 N1/T2 N1 & $0.6(33)$ & 255 & $51-\mathrm{AWD}$ \\
$5(63, \mathrm{M})$ & $32 / 7$ & Barrett/Tis & $3.2(106)$ & 325 & $36-$ AWD \\
\hline
\end{tabular}

$I T$, Distance from incisors in flexible esophagoscopy; $u$, endoscopic ultrasound; $p$, postoperative; Barrett, Barrett esophagus; Tis, carcinoma in situ; $\mathrm{FEV}_{1}$, forced expiratory volume in 1 second (liter and \%); $A W D$, alive without disease; $D W D$, dead without disease. 